\title{
Green light reduces elongation when partially replacing sole blue light independently from cryptochrome 1a
}

\author{
Xue Zhang $^{1,2}$ (1) | Mehdi Bisbis ${ }^{2,3}$ | Ep Heuvelink ${ }^{2}$ | Weijie Jiang ${ }^{1}$ | \\ Leo F. M. Marcelis ${ }^{2}$
}

${ }^{1}$ Key Laboratory of Horticultural Crops Genetic Improvement (Ministry of Agriculture), Institute of Vegetables and Flowers, Chinese Academy of Agricultural Sciences, Beijing, China

${ }^{2}$ Horticulture and Product Physiology Group, Wageningen University, Wageningen, The Netherlands

${ }^{3}$ Leibnitz Institute for Vegetable and Ornamental Production, Germany

Correspondence

Leo F. M. Marcelis, Horticulture and Product Physiology Group, Wageningen University, PO Box 16, 6700 AA Wageningen, The Netherlands

Email: leo.marcelis@wur.nl

Funding information

National Key Research and Development Program of China, Grant/Award Number: 2019YFD10000300

Edited by E. Pesquet

\begin{abstract}
Although green light is sometimes neglected, it can have several effects on plant growth and development. Green light is probably sensed by cryptochromes (crys), one of the blue light photoreceptor families. The aim of this study is to investigate the possible interaction between green and blue light and the involvement of crys in the green light response of plant photomorphogenesis. We hypothesize that green light effects on morphology only occur when crys are activated by the presence of blue light. Wild-type Moneymaker (MM), cry1a mutant (cry1a), and two CRY2 overexpressing transgenic lines (CRY2-OX3 and CRY2-OX8) of tomato (Solanum lycopersicum) were grown in a climate chamber without or with green light $\left(30 \mu \mathrm{mol} \mathrm{m} \mathrm{m}^{-2} \mathrm{~s}^{-1}\right)$ on backgrounds of sole red, sole blue and red/blue mixture, with all treatments having the same photosynthetic photon flux density of $150 \mu \mathrm{mol} \mathrm{m} \mathrm{m}^{-2} \mathrm{~s}^{-1}$. Green light showed no significant effects on biomass accumulation, nor on leaf characteristics such as leaf area, specific leaf area, and chlorophyll content. However, in all genotypes, green light significantly decreased stem length on a sole blue background, whereas green light hardly affected stem length on sole red and red/blue mixture background. MM, cry1a, and CRY2-OX3/8 plants all exhibited similar responses of stem elongation to green light, indicating that cry1a, and probably cry2, is not involved in this green light effect. We conclude that partially replacing blue light by green light reduces elongation and that this is independent of cry $1 \mathrm{a}$.
\end{abstract}

\section{1 | INTRODUCTION}

Leaves reflect a relatively large part of green light (G), causing the green appearance of plants. Green light was for a long time thought to be irrelevant for plant functioning. However, this perception is now fading (Smith et al. 2017). Although leaves appear green, the fraction of green light that is reflected is only about 10-15\% (Paradiso et al. 2011; Smith 1986), while the major share (about $75-80 \%$ ) is absorbed, and the rest transmitted. This suggests that there might very well be a role of green light in photomorphogenesis. Green light may play a major role in controlling plant development in orchestration with red light $(R)$ and blue light $(B)$
(Folta \& Maruhnich 2007). Wang and Folta (2013) suggested that this role is particularly important at low light conditions, like a canopy with a high planting density. On the other hand, Terashima et al. (2009) reported that at high photosynthetic photon flux density (PPFD), G drives leaf photosynthesis more efficiently than $\mathrm{R}$ and $\mathrm{B}$. This is related to the fact that $\mathrm{G}$ can penetrate deep into the mesophyll layers at the single-leaf level (Smith et al. 2017).

There is increasing evidence for the ability of green light to regulate plant photomorphogenesis. Supplementing $G$ to white light (W) or a mixture of $\mathrm{R}$ and $\mathrm{B}(\mathrm{RB})$ increased hypocotyl and petiole length in Arabidopsis (Folta 2004; Wang et al. 2015; Zhang et al. 2011) 
Hypocotyls were longer when G:B ratio was higher (Sellaro et al. 2010). Higher $G$ intensity also increased the content of photosynthetic pigments in Arabidopsis seedlings, and biomass and photosynthetic parameters in leaves of lettuce (Efimova et al. 2013; Golovatskaya \& Karnachuk 2008; Johkan et al. 2012; Muneer et al. 2014). Lettuce plants grown in a mixture of $R, B$ and $G$ (RBG) had larger specific leaf area (SLA) but lower stomatal conductance compared with RB alone, where the total light intensity of RBG was higher than that of RB (Kim 2005). Plant height and dry weight increased in cucumbers when adding $520 \mathrm{~nm}$ G to a mixture of $B, R$ and far-red light (RBFrG) compared with RBFr alone of similar light intensity, whereas such effects were not found when adding $595 \mathrm{~nm}$ G (Brazaityte et al. 2009). In a recent review on green light, Battle et al. (2020) indicated that short-wavelength green light (500$530 \mathrm{~nm}$ ) may lead to different responses compared to long-wavelength green light (530-600 nm). Growing lettuce plants at different combinations of $\mathrm{G}$ with RB showed that growth increased when the fraction of green light was raised from 0 to $24 \%$, but increasing its proportion from 24 to $86 \%$ decreased the growth of leaf area and shoot mass (Dougher \& Bugbee 2001; Kim et al. 2004).

The nature of the green light receptor remains controversial, although most researchers proposed that green light is sensed by cryptochromes (crys) (Banerjee et al. 2007; Bouly et al. 2007; Sato et al. 2015). In higher plants, three crys have been described to date: CRY1 and CRY2, both localized predominantly in the nucleus and the cytoplasm (Lin \& Shalitin 2003), and CRY3 in the organelles (Kleine et al. 2003). Two CRY1 (CRY1a and CRY1b), one CRY2 and one CRY3 (CRY-DASH) genes have been isolated in tomato (Facella et al. 2006; Perrotta et al. 2000, 2001). It has been suggested that green light reverses the action of blue light on the activity of crys, making them inactive for blue light (Banerjee et al. 2007; Bouly et al. 2007). This antagonistic blue-green interaction was supposed to be mediated through the interconversion of flavin redox states of crys. The authors concluded that the fully oxidized chromophore (FAD) absorbs blue light and is then converted to a semi-reduced chromophore (FADH), which is the biologically active green-absorbing form. However, there are some inconsistencies with this proposition. Wang et al. (2013) found that $G$ cannot reverse the cry-mediated $B$ inhibition of early stem elongation but acts additively with $B$ to drive cry-mediated inhibition. Sato et al. (2015) found that sole G or sole B during the night period inhibited hypocotyl elongation, which seemed to be mediated by cry2. The carotenoid zeaxanthin has been suggested as a photoreceptor for the stomatal blue light response, which could be reversed when adding $G$ to $B$, indicating that zeaxanthin might absorb $G$ (Frechilla et al. 1999, 2000). Using different photoreceptor mutants of Arabidopsis, Zhang et al. (2011) concluded that the increased leaf inclination and petiole length induced by supplemental $G$ to RB was mediated neither by crys nor by phytochrome A (phyA) and B (Zhang et al. 2011). A yet unknown green light photoreceptor may exist in plants. While the cryptochrome family has been well studied in the model plant Arabidopsis, information about the crys is limited in crop plants, such as tomato, that has an architecture very different from that of Arabidopsis (Fantini et al. 2019; Liu et al. 2018).
The aim of this study is to investigate the interaction between $G$ and $B$ and the involvement of crys in the green light response of plant photomorphogenesis. We hypothesize that the effect of $G$ on stem elongation only occurs when crys are activated by the presence of B. Experiments in climate rooms were conducted where the effects of $525 \mathrm{~nm} \mathrm{G}$ were studied by replacing $20 \%$ background light of sole $B$, sole $\mathrm{R}$ as well as red/blue mixture. To study the involvement of crys, we used a cryptochrome-deficient genotype and two genotypes overexpressing crys. In contrast to many other studies on G, we kept the PPFD as well as the ratio of other colors the same when $G$ was added.

\section{2 | MATERIALS AND METHODS}

\section{1 | Plant materials and growth conditions}

Tomato (Solanum lycopersicum) seeds of wild-type Moneymaker (MM) and two CRY2 overexpressing transgenic lines (CRY2-OX3 and CRY2-OX8, previously named line 52.3 and line 52.8 in Giliberto et al. 2005) were kindly provided by Dr. Elio Fantini, ENEA Trisaia Research Center, Italy. Tomato cry1a mutant seeds were obtained from Tomato Genetic Resource Center, UC Davis, USA. Seeds were germinated in vermiculite under darkness for 3 days and then transferred to $150 \mu \mathrm{mol} \mathrm{m}{ }^{-2} \mathrm{~s}^{-1}$ white light-emitting diode (LED; GreenPower, Phillips). Day/night temperature was maintained at $22 / 18^{\circ} \mathrm{C}$ with a photoperiod of $18 \mathrm{~h}$. Relative air humidity was $70 \%$.

Ten days after sowing, plants were transplanted in $11 \times 11 \times 12 \mathrm{~cm}$ black plastic pots filled with $\sim 6 \mathrm{~mm}$ expanded clay grid (4-8 $\mathrm{mm}$; Jongkind hydrocorns) and light treatments started. The treatments consisted of sole blue, sole red, red/blue mixture (red/blue ratio $=3 / 1$ ) with or without green. Total PPFD was kept at $150 \mu \mathrm{mol} \mathrm{m} \mathrm{m}^{-2} \mathrm{~s}^{-1}$ at the top of plants in all treatments. When green was added, the red/blue ratio was kept the same as in the treatment without green light (Table 1). Light was provided by narrow band LEDs with peaks at $447 \mathrm{~nm}$ (blue; Greenpower, Philips), 667 nm (red; Greenpower, Philips), and 525 nm (green; Lumileds; Figure S1). PPFD, phytochrome photostationary state (PSS; Sager et al. 1986), and the fraction of red (600-700 nm), blue (400$500 \mathrm{~nm}$ ), and green (500-600 nm) light in all LED treatments were measured by an Apogee Spectroradiometer SS-110. These measurements were performed at a $45 \mathrm{~cm}$ distance from the light source, and the light source was kept at a $40 \sim 50 \mathrm{~cm}$ distance from the top of plants by changing the height of the LEDs twice a week during the growing period. The top of plants of different genotypes was kept at the same level by adjusting the height of pots.

\section{$2.2 \mid$ Measurements}

Plants were measured 21 days after transplanting. Stem length was measured up to the apex. Total leaf area was measured using 
TAB LE 1 Total PPFD (photosynthetic photon flux density) and PPFD of red (R; 600-700 nm), blue (B; 400-500 nm), and green (G; 500-600 $\mathrm{nm}$ ) for the six spectral treatments as well as the phytochrome photostationary state (PSS)

\begin{tabular}{|c|c|c|c|c|c|}
\hline Spectral treatment & \multicolumn{4}{|c|}{ Light intensity ( $\mu \mathrm{mol} \mathrm{m} \mathrm{m}^{-2} \mathrm{~s}^{-1}$ ) } & PSS \\
\hline $\mathrm{R}$ & 150 & 150 & & & 0.880 \\
\hline RG & 150 & 120 & & 30 & 0.884 \\
\hline B & 150 & & 150 & & 0.505 \\
\hline BG & 150 & & 120 & 30 & 0.578 \\
\hline RBG & 150 & 90 & 30 & 30 & 0.877 \\
\hline
\end{tabular}

a leaf area meter (model LI-3000; LI-COR). Roots, stems and leaves were separated and dried in a ventilated oven at $105^{\circ} \mathrm{C}$ for $24 \mathrm{~h}$ to determine the dry weight (DW). From the above, the specific leaf area $\left(\mathrm{m}^{2}\right.$ of leaf area $\mathrm{g}^{-1}$ of leaf $\left.\mathrm{DW}\right)$ was determined.

The fourth leaf counted from the top was used for measuring photosynthetic pigments. Photosynthetic pigments of fresh leaves were extracted in 100\% N,N-Dimethylformamide (DMF) and then measured using Varian Cary 4000 spectrophotometer. The equations of Wellburn (1994) were used to determine concentrations of chlorophyll $a$ (Chla) and $b$ (Chlb) as well as total carotenoids (Car) in $\mu \mathrm{g}$ $\mathrm{ml}^{-1}$ DMF.

$$
\begin{aligned}
\text { Chla } & =12 \mathrm{~A}_{663.8}-3.11 \mathrm{~A}_{646.8} \mathrm{Chlb}=20.78 \mathrm{~A}_{646.8}-4.88 \mathrm{~A}_{663.8} \mathrm{Car} \\
& =\left(1000 \mathrm{~A}_{480}-1.12 \mathrm{Chla}-34.07 \mathrm{Chlb}\right) / 245
\end{aligned}
$$

\section{3 $\quad$ Statistical set-up and analysis}

The experiment was conducted five times after each other, representing five blocks. Six light treatments were applied to four genotypes. In each block, measurements were performed on three individual plants for each combination of light treatment and genotype (nine plants for stem length and leaf number); hence the total number of replicate plants per combination of light treatment and genotype were 15 (45 for stem length and leaf number). A split-plot design was applied to each block, with light treatment as the whole-plot factor and genotype as the subplot factor. Analysis of variance (ANOVA) was conducted using Genstat 19.0 for Windows. Residuals were tested for normality (Sapiro-Wilk test at $p=0.05$ ). In the case of non-normal residuals, the original data were log-transformed, which always resulted in normal residuals. For mean separation, Fisher's unprotected LSD test at $p=0.05$ was used; "unprotected" because we also applied this test for testing differences among individual combinations of light treatment $\times$ genotype when the $F$-test for interaction was not significant at $p=0.05$.

\section{3 | RESULTS}

\section{1 | Green light reduced stem length when partially replacing sole blue light}

Figure 1 shows the pictures of representative plants of the four genotypes grown under the six light treatments. Partially (20\%) replacing sole $B$ by $G(B G)$ significantly reduced stem length in all four genotypes (Figure 2). Partially replacing RB by G (RBG) did not change stem length in any of the genotypes. Partially replacing sole $R$ by $G$ (RG) did not affect stem length in the wild-type MM and cry1a mutant but slightly reduced stem length in the two $C R Y 2$ overexpressors CRY2-OX3 and CRY2-OX8 (Figure 2). Interestingly, CRY1a-deficient plants were remarkably taller than other genotypes, even under the $100 \% \mathrm{R}$ background (Figure 2).

\subsection{Green light did not induce changes in specific leaf area but reduced shoot:root ratio of CRY2 overexpression lines}

No significant interaction between light treatment and genotype was observed in SLA (Figure S3). Partially replacing sole blue and red/blue mixture by green light did not significantly change the leaf area, except that BG remarkably reduced leaf area of CRY2-OX8 compared to $B$ (Figure 3). However, partially replacing sole $R$ by $G$ increased leaf area, though this was only significant in cry1a mutant and CRY2-OX3 (Figure 3). The CRY2-OX3 and CRY2-OX8 plants tended to have less leaf area than MM and cry1a mutant in all light treatments, though it was only significant when the background light contained blue light (B, BG, RB, and RBG).

The shoot:root ratio of both wild-type and cry1a mutant did not respond to green light (Figure 4A,B). However, $G$ significantly reduced the shoot: root ratio of $C R Y 2$ overexpressors under sole $B$ background, as well as that of CRY2-OX3 under sole R (Figure 4C,D). In line with the results of stem length (Figure 2), cry1a mutant had the highest shoot: root ratios, though the effects were only significant under B and BG (Figure 4). 
$\mathrm{R}$
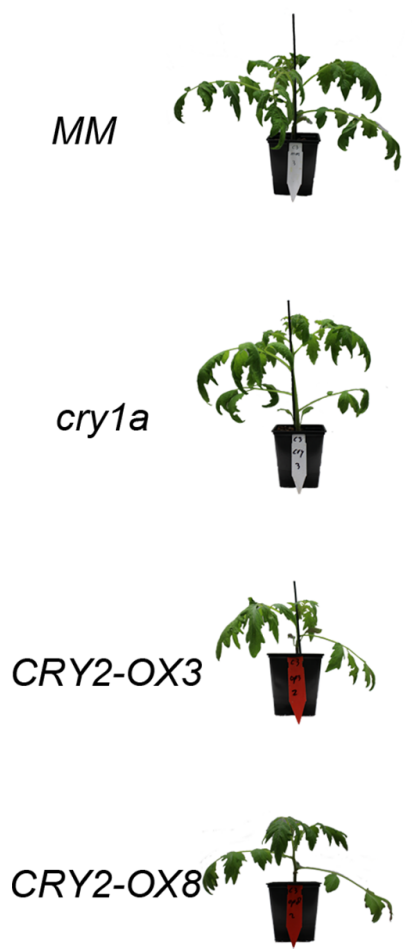

RG
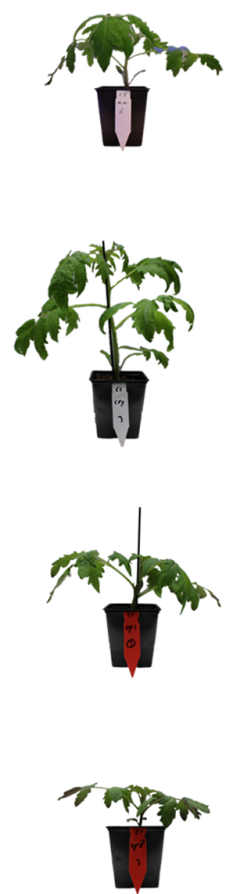

B
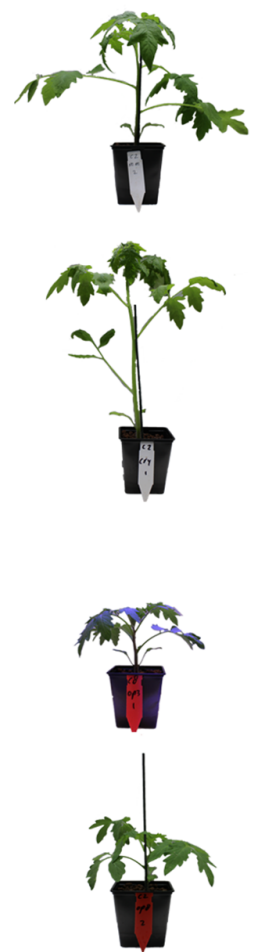

BG
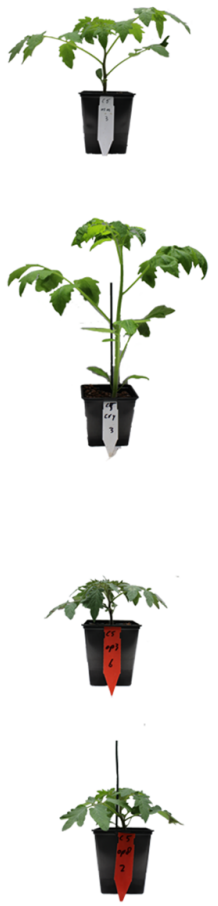
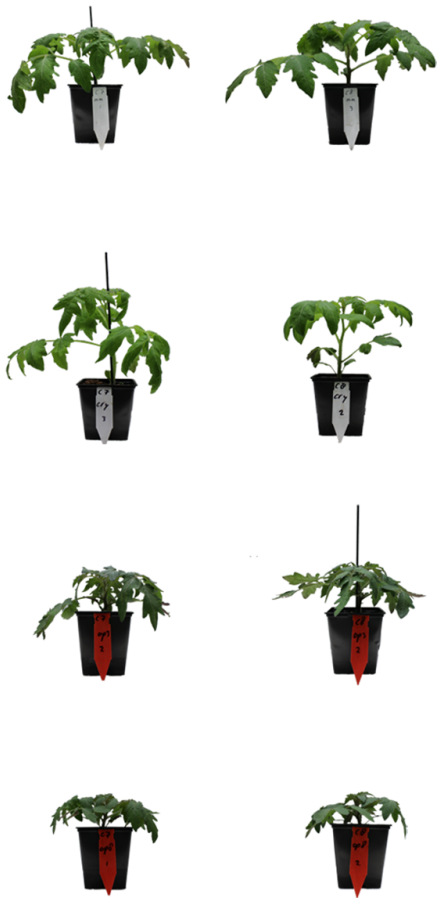

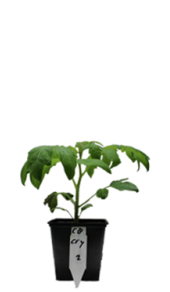

RBG
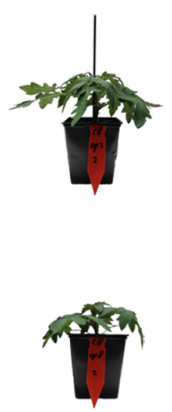

FIGURE 1 Effect of partially (20\%) replacing sole red (R), sole blue (B) or red/blue (RB; ratio 3:1) by green (G) light on the phenotypes of four tomato genotypes: MM (Moneymaker, wild-type), cry1a (CRY1a-deficient), CRY2-OX3 (CRY2 overexpressing, line 52.3), and CRY2-OX8 (CRY2 overexpressing, line 52.8). A picture of one representative plant is shown per combination of light treatment and genotype

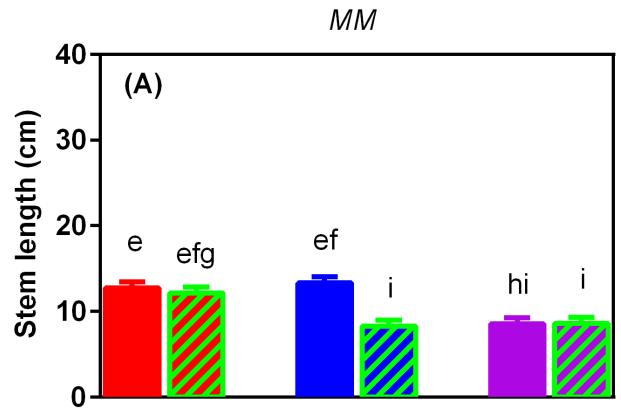

CRY2-OX3

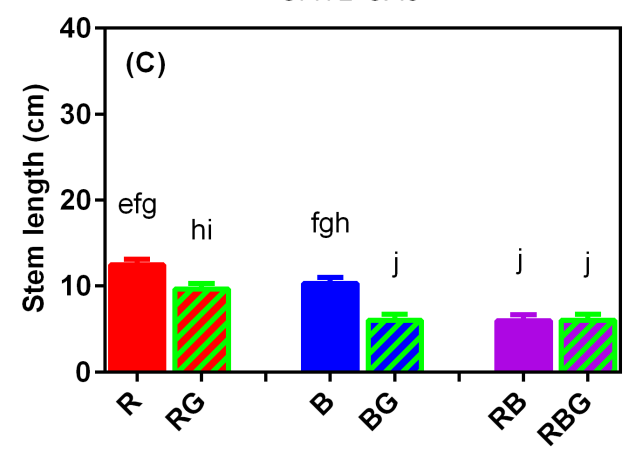

light treatment
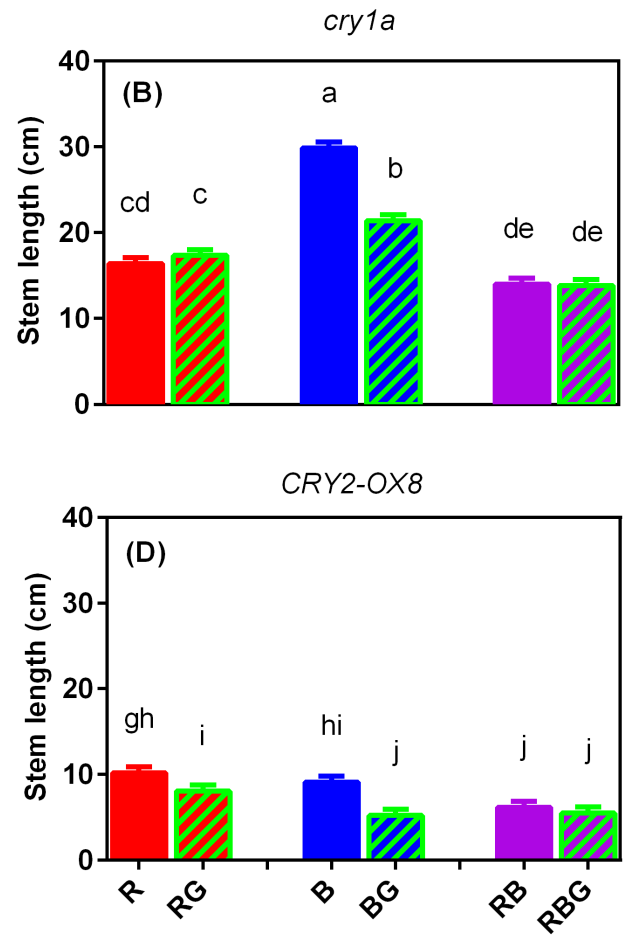

light treatment
IGURE 2 F Effect of partially (20\%) replacing sole red (R), sole blue (B) or red/blue (RB; ratio 3:1) by green ( $G$ ) light on stem length on day 21 after transplanting of four tomato genotypes, (A) MM (Moneymaker, wild-type), (B) cry1a (CRY1a-deficient), (C) CRY2-OX3 (CRY2 overexpressing, line 52.3), and (D) CRY2-OX8 (CRY2 overexpressing, line 52.8).

There was a significant interaction between light treatment and genotype (logtransformed data; $p<0.001$ ). Different letters above bars indicate significant differences among light treatment $\times$ genotype combinations ( $p=0.05$ ), thus it allows comparison of bars among figures A-D. Vertical bars indicate SE of the mean of five blocks ( $n=5$ ), each based on nine replicate plants 
FIGURE 3 Effect of partially (20\%) replacing sole red $(R)$, sole blue (B) or red/blue (RB; ratio $3: 1$ ) by green $(G)$ light on leaf area on day 21 after transplanting of four tomato genotypes, (A) MM

(Moneymaker, wild-type), (B) cry1a (CRY1a-deficient), (C)

CRY2-OX3 (CRY2 overexpressing, line 52.3), and (D) CRY2-OX8 (CRY2 overexpressing, line 52.8). Interaction between light treatment and genotype was significant (log-transformed data; $p$ < 0.001). Different letters above bars indicate significant differences between light treatments $\times$ genotype combinations ( $p=0.05$ ), thus it allows comparison of bars among figures A-D. Vertical bars indicate $S E$ of the mean of five blocks ( $n=5)$, each based on three replicate plants

FIGURE 4 Effect of partially (20\%) replacing sole red $(R)$, sole blue (B) or red/blue (RB; ratio 3:1) by green $(G)$ light on shoot: Root ratio on day 21 after transplanting of four tomato genotypes, (A) MM

(Moneymaker, wild-type), (B) cry1a (CRY1a-deficient), (C) CRY2-OX3 (CRY2 overexpressing, line 52.3), and (D) CRY2-OX8 (CRY2 overexpressing, line 52.8). A significant interaction between light treatment and genotype was observed ( $p=0.013$ ). Different letters above bars indicate significant differences between light treatment $\times$ genotype combinations ( $p=0.05)$, thus it allows comparison of bars among figures A-D. vertical bars indicate $S E$ of the mean of five blocks $(n=5)$, each based on three replicate plants
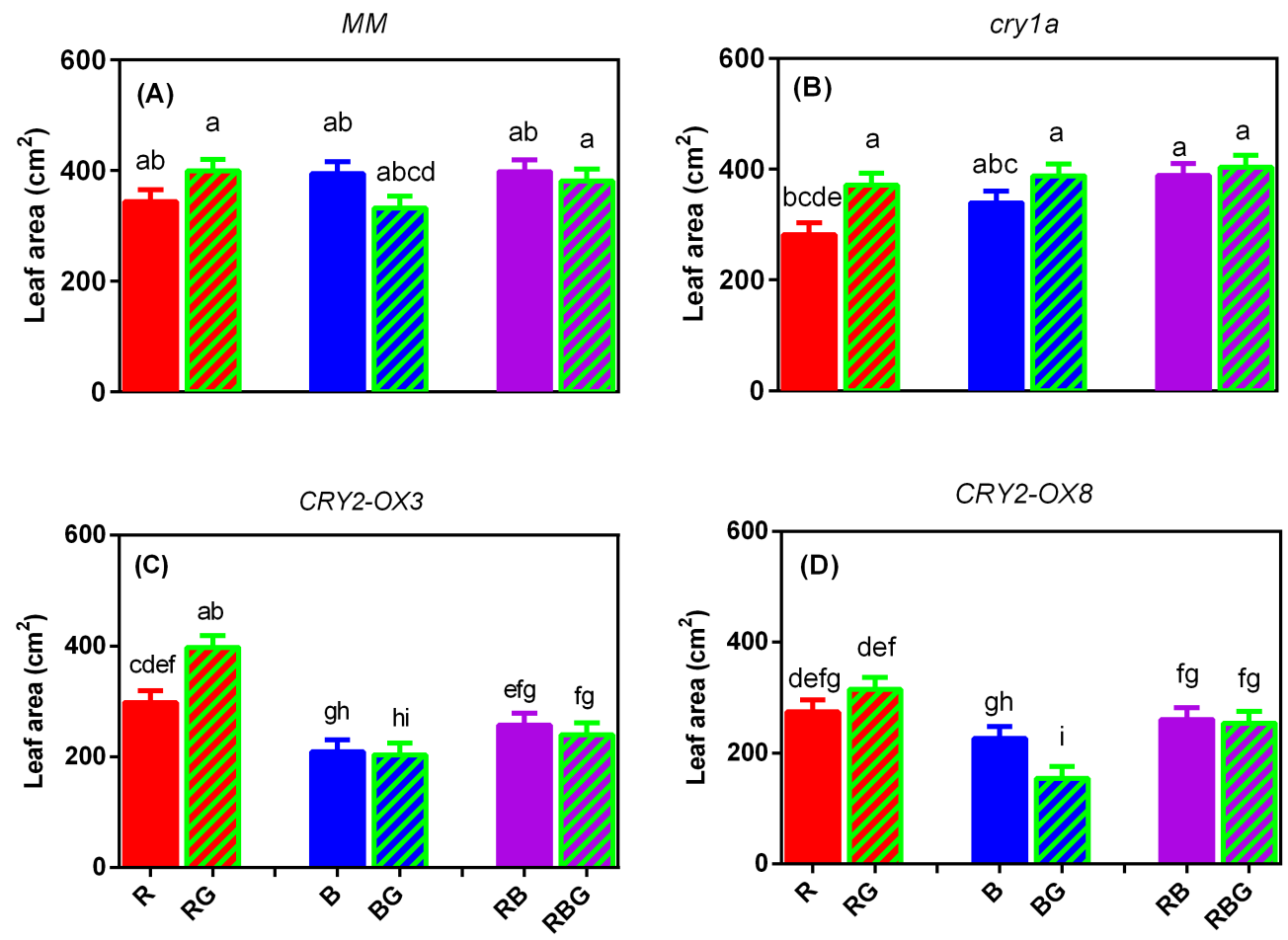

light treatment
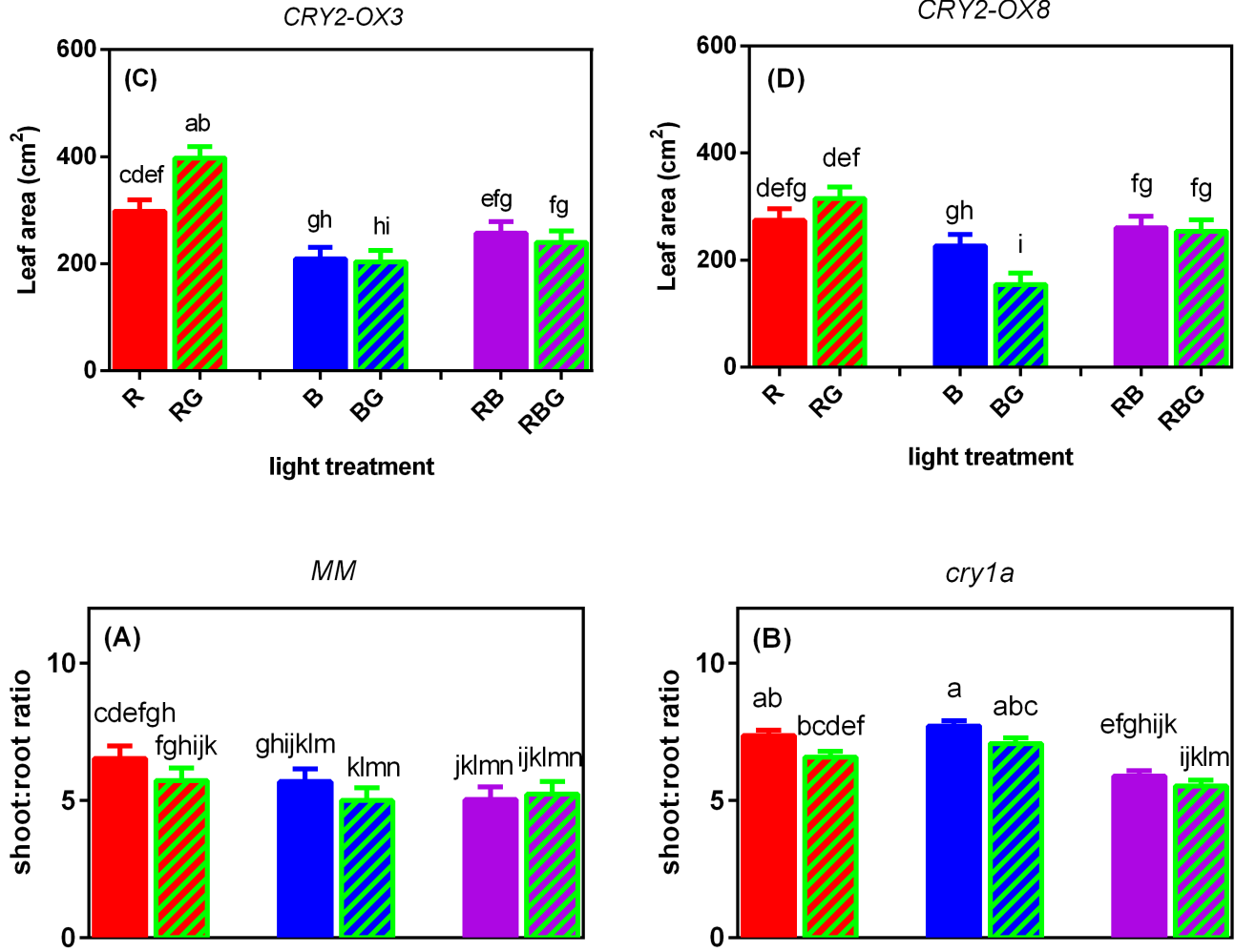

light treatment

cry1a

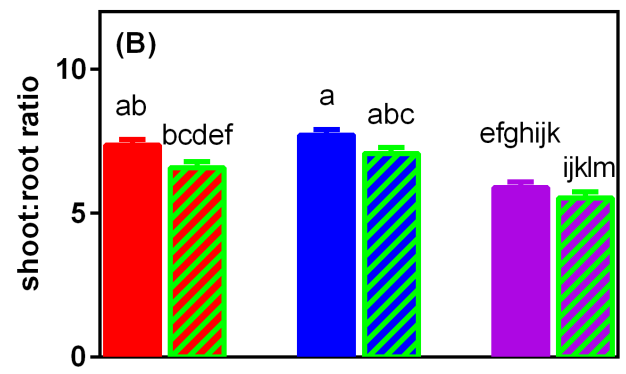

CRY2-OX8

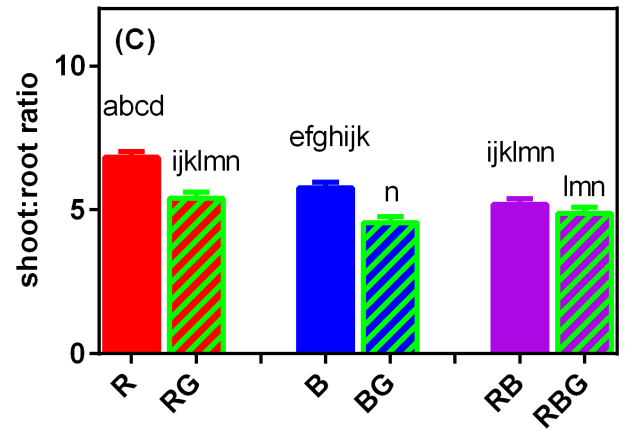

light treatment

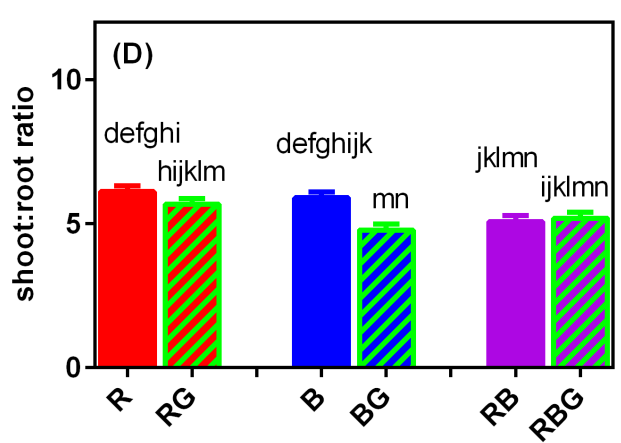

light treatment

\subsection{No significant effect of green light on biomass accumulation}

The total dry weight was not significantly affected by partially replacing the different colors $(R, B$, or $R B)$ by green light, nor was there a significant difference among the genotypes and other spectra (Figure 5). Similarly, the contents of chlorophylls (chls, chl $a+b$ ) and total carotenoids (car), as well as the ratio of chl $a$ to chl $b$ and $\mathrm{chl} a+b /$ car ratio were mostly not influenced by the genotypes and light treatments (Figure S4). However, partially replacing sole $R$ 

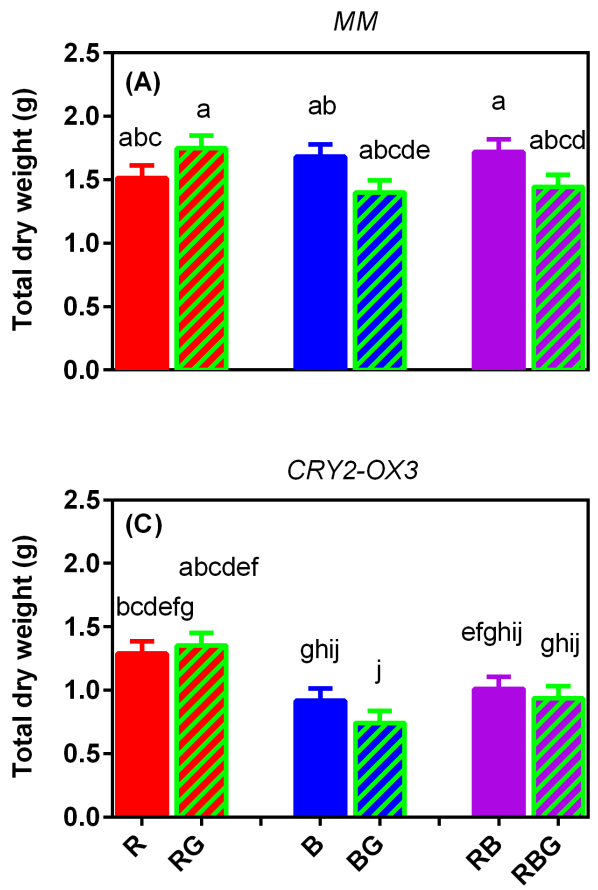

light treatment
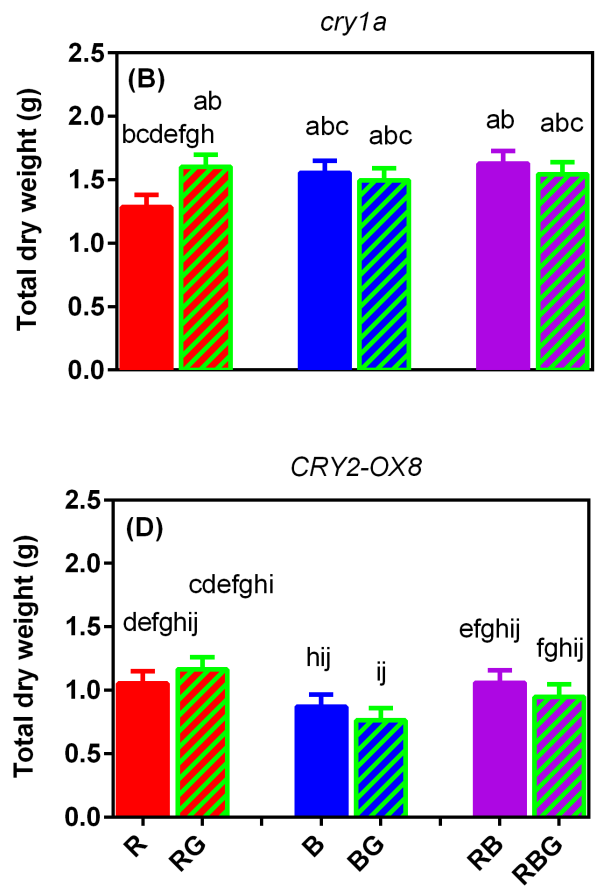

light treatment

FI GURE 5 Effect of partially (20\%) replacing sole red (R), sole blue (B) or red/blue (RB; ratio 3:1) by green (G) light on total dry weight on day 21 after transplanting of four tomato genotypes, (A) MM (Moneymaker, wild-type), (B) cry1a (CRY1a-deficient), (C) CRY2-OX3 (CRY2 overexpressing, line 52.3), and (D) CRY2-OX8 (CRY2 overexpressing, line 52.8). No significant interaction between light treatment and genotype was found $(p=0.686)$, but the effects of light treatment $(p=0.04)$ and genotype $(p<0.001)$ were significant. Different letters above bars indicate significant differences between light treatment $\times$ genotype combinations $(p=0.05)$, thus it allows comparison of bars among figures $A-$ D. Vertical bars indicate SE of the mean of five blocks $(n=5)$, each based on three replicate plants

by $\mathrm{G}$ significantly increased the chl $a+b /$ car ratio of the cry1a mutant.

\section{4 | DISCUSSION}

\section{1 | Partially replacing sole blue light by green light reduced elongation independent of cry1a}

Tomato stem length was significantly reduced by partially $(20 \%)$ replacing sole blue light by green light, whereas partially replacing sole red or red/blue mixture with $\mathrm{G}$ had little effect on stem length (Figure 2). These effects were due to the elongation of internodes as leaf number was not affected (Figure S2). CRYs were reported to mediate hypocotyl elongation inhibition driven by sole blue light or sole green light compared to darkness in Arabidopsis, and $\mathrm{G}$ acts additively with $B$ to drive cryptochrome-mediated inhibition of elongation (Wang et al. 2013). CRY1a-deficient and CRY2 overexpressing lines (CRY2 OX3 and CRY2-OX8) showed similar responses of stem length to partially replacing sole $B$ by $G$ than the wild-type MM (Figure 2). Hence, our results indicated that this green light response was independent of cry1a, probably independent of cry2 as well.

The stem length of the cry $1 a$ mutant was remarkably longer than the other genotypes under the same light treatment (Figure 2), confirming the involvement of CRY1a in the inhibition of internode elongation (Ninu et al. 1999). The overexpression of CRY2 in CRY2OX3 and CRY2-OX8 induced shorter stems (Figure 2), also confirming the involvement of cry2 under all light treatments (Yang et al. 2017).

Through blue light, the neutral FAD chromophore in crys is converted into an active state (FADH) absorbing green light, which converts the crys into a fully reduced and inactive state (Lin \& Shalitin 2003; Banerjee et al. 2007; Bouly et al. 2007). Green light partially inhibits cry2 oxidation by blue light (Banerjee et al. 2007; Bouly et al. 2007; Frechilla et al. 2000; Zeugner et al. 2005), contributing to reduced levels of FADH. However, this photocycle model could not explain all interactions between blue and green light on stem length, like the finding that $G$ could also act additively to B to inhibit cry-mediated stem elongation in Arabidopsis (Wang et al. 2013). In contrast to several other studies on the role of crys, where Arabidopsis seedlings (including cry-null mutants) received light for very short periods (e.g. $30 \mathrm{~min}$ in the works of Banerjee et al. and Bouly et al. 2007), our study was conducted with larger tomato plants that were exposed to different light spectra for a number of weeks. In such long-term experiments, the responses of the measured parameters (leaf expansion, stem growth, etc.) can be under the control of many photoreceptors and many cellular pathways (Hammad et al. 2020). Therefore, apart from the direct effects of green light on crys, indirect effects can also play a role. In our study $\mathrm{G}$ induced a similar response of stem elongation in $C R Y 2$ overexpressing lines than 
in the wild-type (Figure 2), confirming that the mechanism underlying crys activation during plant growth has not been elucidated.

Another interpretation of the G-reduced elongation when partially replacing sole $B$ is that $G$ may activate phytochromes, as also suggested by the increase in PSS value (Table 1). Partially replacing sole $\mathrm{B}$ by $\mathrm{R}$ also remarkably reduced elongation (Figures 1 and 2), suggesting a potent cry-phy interaction. Battle et al. (2020) summarized the reported interactions between blue and green light, indicating that green light could act to complement or antagonize blue lightinduced responses dependent on the wavelength of the green light, either through the direct repression of cryptochrome signaling or via a phytochrome-dependent mechanism.

\subsection{The involvement of CRY2 in regulating plant photomorphogenesis}

In contrast with $M M$ and cry $1 a$ mutant, stem length was reduced in CRY2-OX3 and CRY2-OX8 when partly replacing sole $\mathrm{R}$ by $\mathrm{G}$ (Figure 2 ), while partly replacing $B$ by $G$ induced a lower shoot: root ratio and smaller leaf area (not significant in CRY2-OX3; Figures 3 and 4). These results indicate the involvement of $C R Y 2$ in green light effects on stem length, shoot: root ratio and leaf area. However, it is not easy to interpret why $G$ did not affect the stem length of CRY2 overexpressors when partially replacing RB mixture. Maybe this effect was absent because these genotypes had quite short stems when grown under $\mathrm{RB}$ compared to sole $\mathrm{R}$ or $\mathrm{B}$.

Comparing the tomato CRY2 overexpressing lines with wild-type plants, CRY2 may control vegetative development and photosynthesis as suggested by high-throughput transcriptomic and proteomic analyses by Lopez et al. (2012), and by the overproduction of chlorophylls in CRY2 overexpressors (Giliberto et al. 2005). However, we did not observe significant differences in SLA and chlorophyll content between CRY2-OX3/OX8 and MM (Figures S3 and S4). We conclude that the effects of $C R Y 2$ on phenotype are limited, which might result from its redundant role with $C R Y 1 a$.

\subsection{PHYs play a role in blue light effects on elongation}

Besides mediation by $C R Y s$, the blue light effects might also be mediated by PHYs. The PSS value, which is an indicator of phytochrome status, was lower under sole blue than that under all other light treatments; green light had little effect on the PSS value (Table 1). CRYS and PHYs converge blue and red light signals at different levels to co-regulate physiological responses, such as root greening, de-etiolation, shade avoidance symptoms, photoperiodic flowering, etc (Su et al. 2017). Although many studies report that an increasing fraction of blue light reduces stem length (e.g. Kalaitzoglou et al. 2021) due to the involvement of crys, stems under sole $B$ were not the shortest (Figure 2). This may suggest a possible role for phytochrome action. In the cry1a mutant, the effects of blue light on elongation via crys are expected to be minor. Hence, the blue effects on stem elongation in this mutant are mainly mediated by phys, resulting in the tallest plants under sole blue light.

Strikingly, similar to $100 \% \mathrm{~B}, 100 \% \mathrm{R}$ also induced significantly longer cry $1 a$ mutant plants compared to $M M$ (Figure 2), consistent with the results of Fantini et al. (2019). On the contrary, Ninu et al. (1999) found that 8 days old CRY1a antisense tomato plants did not show an elongated hypocotyl under red light but under blue light (both approximately $8 \mu \mathrm{mol} \mathrm{m}{ }^{-2} \mathrm{~s}^{-1}$ ). These differences in results might be caused by the fact that the CRY1a gene is not knocked out but only downregulated in CRY1a antisense plants, or by differences in the development stage or light intensity. Accumulating evidence in the model plant Arabidopsis has revealed that CRYs and PHYs share two mechanistically distinct pathways that coordinately regulate transcriptional changes in response to light. However, the role of photoreceptor interactions and the mechanism responsible for the direct convergence of CRYS and PHYS signals on the COP1/SPA complex or phytochrome-interacting factors (PIFs) remain elusive (Su et al. 2017).

In tomato, cryptochrome 1, phytochromes A, B1, and B2 are all capable of mediating responses to $B$ under some circumstances (Weller et al. 2001). In Arabidopsis, CRYs may act in a blue-light independent manner to affect $P H Y$ regulation of gene expression and development, resulting in different protein expression between the WT and cry1cry2 mutant in red light as well as in blue light (Lopez et al. 2012; Yang et al. 2008). Arabidopsis CRY1 interacts directly with PIF4 in a blue light-dependent manner to repress the transcription activity of PIF4 (Ma et al. 2016). This indicates that stem elongation in cry $1 a$ mutants under sole $\mathrm{R}$ could be mediated by downstream genes shared by CRYs and PHYs (Facella et al. 2012; Su et al. 2017). However, the extent and relative importance of their individual contributions differ depending on irradiance, which other photoreceptors are present, and which plant process is examined.

\section{4 | Replacing $20 \%$ of red, red/blue, or blue light by green had no significant effect on biomass production}

McCree (1972) measured the instantaneous response of leaf photosynthesis to different spectra, finding that the quantum yield of photosynthesis of green photons $(525 \mathrm{~nm}$ ) can be about $25-30 \%$ less than that of red photons $(675 \mathrm{~nm})$, while the quantum yield of green is comparable to that of blue photons $(450 \mathrm{~nm})$. However, this may not be representative of whole plants or plant communities grown at high PPFD under mixed colors of light. Green light could drive carbon fixation deep within leaves (Sun et al. 1998), even more efficiently than R or B (Nishio 2000), because it could penetrate deep into the mesophyll layers (Smith et al. 2017). In our study, where the light contained 0 or $20 \%$ green, the plant biomass production rate was not significantly affected by green light (Figure 5). Similarly, the contents of chlorophyll $a$ and $b$ and carotenoids, as well as their ratios, were hardly affected by green light (Figure S4).

Partially replacing sole $\mathrm{R}$ or $\mathrm{B}$ or $\mathrm{R} / \mathrm{B}$ mixture by green light did not cause differences in leaf area, SLA, shoot: root ratio and biomass 
of MM and cry1a mutant. This contradicts previous findings on green light responses, but in those studies PPFD also increased when adding G (Kim 2005; Novičkovas et al. 2012; Samuolienè et al. 2012). Zhang et al. (2011) reported that $40 \%$ green light induced a shade avoidance response in Arabidopsis seedlings, whereas $10 \%$ did not. Too much G (51\%) or too little $(0 \%)$ decreased lettuce growth, while about $24 \%$ resulted in the highest growth rate (Kim et al. 2004). However, in our study, $20 \%$ G did not induce such effects, which is comparable to the study of Hernández and Kubota (2015), who analyzed the effect of $28 \%$ G in cucumber. Kaiser et al. (2019) found that replacing $32 \%$ of a red/blue mixture spectrum by green light significantly increased plant biomass and yield. These different observations among studies suggest that $\mathrm{G}$ effects might be genotype-specific and dependent on and/or interact with other environmental conditions.

Although the effects of light spectrum on biomass production were limited in this study, there were profound effects on plant shoot architecture (e.g. stem length). This can be of practical relevance in horticulture to manipulate shoot architecture.

\section{5 | CONCLUSIONS}

Tomato stem elongation was significantly reduced by green light when it partially replaced sole blue light, which may suggest a role for cryptochrome. However, cry1a mutant and CRY2 overexpressing plants showed similar trends on stem length as the wild-type. This indicates that this response to green light is probably independent of cry1a and cry2. Moreover, cry1a mutant plants were significantly taller than other genotypes under all spectra, whereas CRY2 overexpressing plants had a much shorter stem. We conclude that cry1a, and probably cry2, are not involved in green light effects on elongation under $\mathrm{R}$ and $\mathrm{B}$ background of our study.

\section{ACKNOWLEDGMENTS}

This research was supported by The National Key Research and Development Program of China (2019YFD10000300). We thank Unifarm of Wageningen University and Research (WUR) for producing the tomato seeds and technical help in conducting the experiment. We thank Lumileds for providing green LEDs. We thank the Tomato Genetic Resource Center, University of California, Davis (USA) for providing cry1a mutant seeds. We thank Dr. Elio Fantini, from ENEA Trisaia Research Center, Italy, for providing seeds of wild-type MM and two CRY2 overexpressing lines, and for his critical reading of the manuscript. We thank Wouter Mooij for assistance with sample collection, measurement and data analysis. We thank Arjen van de Peppel for assistance with lab measurement.

\section{AUTHOR CONTRIBUTIONS}

Xue Zhang, Ep Heuvelink, and Leo F. M. Marcelis conceived and designed the experiment. Xue Zhang and Mehdi Bisbis conducted the experiment. Xue Zhang, Mehdi Bisbis and Ep Heuvelink analyzed the data. Xue Zhang, Mehdi Bisbis and Leo F. M. Marcelis interpreted the data. Xue Zhang wrote the first draft of the manuscript, large parts were edited by Leo $\mathrm{F}$.
M. Marcelis and all authors commented on previous versions of the manuscript. All authors read and approved the final manuscript.

\section{DATA AVAILABILITY STATEMENT}

The data that support the findings of this study are available from the corresponding author upon reasonable request.

\section{ORCID}

Xue Zhang (iD) https://orcid.org/0000-0002-7562-0226

\section{REFERENCES}

Banerjee, R., Schleicher, E., Meier, S., Viana, R.M., Pokorny, R., Ahmad, M. et al. (2007) The signaling state of Arabidopsis cryptochrome 2 contains flavin semiquinone. The Journal of Biological Chemistry, 282, 14916-14922.

Battle, M.W., Vegliani, F. \& Jones, M.A. (2020) Shades of green: untying the knots of green photoperception. Journal of Experimental Botany, 71, 5764-5770.

Bouly, J.P., Schleicher, E., Dionisio-Sese, M., Vandenbussche, F., Van Der Straeten, D., Bakrim, N. et al. (2007) Cryptochrome blue light photoreceptors are activated through interconversion of flavin redox states. The Journal of Biological Chemistry, 282, 9383-9391.

Brazaitytè, A., Duchovskis, P., Urbonavičiūtè, A., Samuolienè, G., Jankauskienè, J., Aistè Kasiulevičutè-bonakèrè, A. et al. (2009) The effect of light-emitting diodes lighting on cucumber transplants and after-effect on yield. Zemdirbyste-Agriculture, 96, 102-118.

Dougher, T.A.O. \& Bugbee, B. (2001) Evidence for yellow light suppression of lettuce growth. Photochemistry and Photobiology, 73, 208-212.

Efimova, M.V., Karnachuk, R.A., Kusnetsov, V.V. \& Kuznetsov, V.V. (2013) Green light regulates plastid gene transcription and stimulates the accumulation of photosynthetic pigments in plants. Doklady Biological Sciences, 451, 253-256.

Facella, P., Daddiego, L. \& Perrotta, G. (2012) CRY1a influences the diurnal transcription of photoreceptor genes in tomato plants after gibberellin treatment. Plant Signaling \& Behavior, 7, 1034-1036.

Facella, P., Lopez, L., Chiappetta, A., Bitonti, M.B., Giuliano, G. \& Perrotta, G. (2006) CRY-DASH gene expression is under the control of the circadian clock machinery in tomato. FEBS Letters, 580, 46184624.

Fantini, E., Sulli, M., Zhang, L., Aprea, G., Jimenez-Gomez, J.M., Bendahmane, A. et al. (2019) Pivotal roles of cryptochromes 1a and 2 in tomato development and physiology. Plant Physiology, 179, 732-748.

Folta, K.M. (2004) Green light stimulates early stem elongation, antagonizing light-mediated growth inhibition. Plant Physiology, 135, 14071416.

Folta, K.M. \& Maruhnich, S.A. (2007) Green light: a signal to slow down or stop. Journal of Experimental Botany, 58, 3099-3111.

Frechilla, S., Talbott, L.D., Bogomolni, R.A. \& Zeiger, E. (2000) Reversal of blue light-stimulated stomatal opening by green light. Plant \& Cell Physiology, 41, 171-176.

Frechilla, S., Zhu, J., Talbott, L.D. \& Zeiger, E. (1999) Stomata from npql, a zeaxanthin-less Arabidopsis mutant, lack a specific response to blue light. Plant \& Cell Physiology, 40, 949-954.

Giliberto, L., Perrotta, G., Pallara, P., Weller, J.L., Fraser, P.D., Bramley, P.M. et al. (2005) Manipulation of the blue light photoreceptor cryptochrome 2 in tomato affects vegetative development, flowering time, and fruit antioxidant content. Plant Physiology, 137, 199-208.

Golovatskaya, I.F. \& Karnachuk, R.A. (2008) Effect of jasmonic acid on morphogenesis and photosynthetic pigment level in Arabidopsis 
seedlings grown under green light. Russian Journal of Plant Physiology, 55, 220-224.

Hammad, M., Albaqami, M., Pooam, M., Kernevez, E., Witczak, J., Ritz, T. et al. (2020) Cryptochrome mediated magnetic sensitivity in arabidopsis occurs independently of light-induced electron transfer to the flavin. Photochemical \& Photobiological Sciences, 19, 341-352.

Hernández, R. \& Kubota, C. (2015) Physiological responses of cucumber seedlings under different blue and red photon flux ratios using LEDs. Environmental and Experimental Botany, 29, 66-74.

Johkan, M., Shoji, K. \& Goto, F. (2012) Effect of green light wavelength and intensity on photomorphogenesis and photosynthesis in Lactuca sativa. Environmental and Experimental Botany, 75, 128-133.

Kaiser, E., Weerheim, K., Schipper, R. \& Dieleman, J.A. (2019) Partial replacement of red and blue by green light increases biomass and yield in tomato. Scientia Horticulturae, 249, 271-279.

Kalaitzoglou, P., Taylor, C., Calders, K., Hogervorst, M. \& Marcelis, L. (2021) Unraveling the effects of blue light in an artificial solar background light on growth of tomato plants. Environmental and Experimental Botany, 184, 104377.

Kim, H.H. (2005) Green-light supplementation for enhanced lettuce growth under red- and blue-light-emitting diodes. HortScience, 39 1617-1622.

Kim, H.H., Goins, G.D., Wheeler, R.M. \& Sager, J.C. (2004) Stomatal conductance of lettuce grown under or exposed to different light qualities. Annals of Botany, 94, 691-697.

Kleine, T., Lockhart, P. \& Batschauer, A. (2003) An Arabidopsis protein closely related to Synechocystis cryptochrome is targeted to organelles. The Plant Journal, 35, 93-103.

Lin, C. \& Shalitin, D. (2003) Cryptochrome structure and signal transduction. Annual Review of Plant Biology, 54, 469-4961.

Liu, C.C., Ahammed, G.J., Wang, G.T., Xu, C.J., Chen, K.S., Zhou, Y.H. et al. (2018) Tomato CRY1a plays a critical role in the regulation of phytohormone homeostasis, plant development, and carotenoid metabolism in fruits. Plant, Cell \& Environment, 41, 354-366.

Lopez, L., Carbone, F., Bianco, L., Giuliano, G., Facella, P. \& Perrotta, G. (2012) Tomato plants overexpressing cryptochrome 2 reveal altered expression of energy and stress-related gene products in response to diurnal cues. Plant, Cell \& Environment, 35(5), 994-1012.

Ma, D., Li, X., Guo, Y., Chu, J., Fang, S., Yan, C. et al. (2016) Cryptochrome 1 interacts with PIF4 to regulate high temperaturemediated hypocotyl elongation in response to blue light. Proceedings of the National Academy of Sciences of the United States of America, 113(1), 224-229.

McCree, K.J. (1972) The action spectrum, absorptance, and quantum yield of photosynthesis in crop plants. Agricultural Meteorology, 9, 191-216.

Muneer, S., Kim, E.J., Park, J.S. \& Lee, J.H. (2014) Influence of green, red and blue light emitting diodes on multiprotein complex proteins and photosynthetic activity under different light intensities in lettuce leaves (Lactuca sativa L.). International Journal of Molecular Sciences, 15, 4657-4670.

Ninu, L., Ahmad, M., Miarelli, C., Cashmore, R.A. \& Giuliano, G. (1999) Cryptochrome 1 controls tomato development in response to blue light. The Plant Journal, 18, 551-556.

Nishio, J.N. (2000) Why are higher plants green? Evolution of the higher plant photosynthetic pigment complement. Plant, Cell \& Environment, 23, 539-548.

Novičkovas, A., Brazaitytè, A., Duchovskis, P., Jankauskienė, J., Samuolienè, G., Virsilè, A. et al. (2012) Solid-state lamps (Leds) for the short-wavelength supplementary lighting in greenhouses: experimental results with cucumber. Acta Horticulturae, 927, 723-730.

Paradiso, R., Meinen, E., Snel, J.F., Visser, D.P., lepern, V.W., Hogewoning, W.S. et al. (2011) Spectral dependence of photosynthesis and light absorptance in single leaves and canopy in rose. Scientia Horticulturae, 127, 548-554.

Perrotta, G., Ninu, L., Flamma, F., Weller, J.L., Kendrick, R.E., Nebuloso, E. et al. (2000) Tomato contains homologues of Arabidopsis cryptochromes1 and 2. Plant Molecular Biology, 42, 765-773.

Perrotta, G., Yahoubyan, G., Nebuloso, E., Renzi, L. \& Giuliano, G. (2001) Tomato and barley contain duplicated copies of cryptochrome 1. Plant, Cell \& Environment, 24, 991-997.

Sager, J.C., Smith, W.O., Edwards, J.L. \& Cyr, K.L. (1986) Use of spectral data to determine photosynthetic efficiency and phytochrome photoequilibria. T ASABE, 31, 1882-1889.

Samuolienè, G., Brazaitytè, A., Duchovskis, P., Virsilè, A., Jankauskienė, J., Sirtautas, R. et al. (2012) Cultivation of vegetable transplants using solid-state lamps for the short-wavelength supplementary lighting in greenhouses. Acta Horticulturae, 952, 885-892.

Sato, M., Nishiuchi, T. \& Sakamoto, T. (2015) Responses to intermittent exposure to green light during the dark period in Arabidopsis thaliana and possible involvement of cryptochrome 2. Plant Biotechnology, 32, 263-266.

Sellaro, R., Crepy, M., Trupkin, S.A., Karayekov, E., Buchovsky, A.S., Rossi, C. et al. (2010) Cryptochrome as a sensor of the blue/green ratio of natural radiation in Arabidopsis. Plant Physiology, 154, 401-409.

Smith, H. (1986) The perception of light quality. In: Kendrick, R.E. \& Kronenberg, G.H.M. (Eds.) Photomorphogenesis in plants. Dordrecht: Springer, pp. 187-217.

Smith, H.L., McAusland, L. \& Murchie, E.H. (2017) Don't ignore the green light: exploring diverse roles in plant processes. Journal of Experimental Botany, 68, 2099-2110.

Su, J., Liu, B., Liao, J., Yang, Z., Lin, C. \& Oka, Y. (2017) Coordination of cryptochrome and phytochrome signals in the regulation of plant light responses. Agronomy, 7, 25.

Sun, J., Nishio, J. \& Vogelmann, T.C. (1998) Green light drives CO2 fixation deep within leaves. Plant \& Cell Physiology, 39, 1020-1026.

Terashima, I., Fujita, T., Inoue, T., Chow, W.S. \& Oguchi, R. (2009) Green light drives leaf photosynthesis more efficiently than red light in strong white light: revisiting the enigmatic question of why leaves are green. Plant \& Cell Physiology, 50, 684-697.

Wang, Y. \& Folta, K.M. (2013) Contributions of green light to plant growth and development. American Journal of Botany, 100, 70-78.

Wang, Y., Maruhnich, S.A., Mageroy, M.H., Justice, J.R. \& Folta, K.M. (2013) Phototropin 1 and cryptochrome action in response to green light in combination with other wavelengths. Planta, 237, 225-237.

Wang, Y., Zhang, T. \& Folta, K.M. (2015) Green light augments far-redlight-induced shade response. Plant Growth Regulation, 77, 147-155.

Wellburn, A.R. (1994) The spectral determination of chlorophylls $a$ and $b$, as well as total carotenoids, using various solvents with spectrophotometers of different resolution. Journal of Plant Physiology, 144, 307-313.

Weller, J.L., Perrotta, G., Schreuder, M.E., Tuinen, A., Koornneef, M., Giuliano, G. et al. (2001) Genetic dissection of blue-light sensing in tomato using mutants deficient in cryptochrome 1 and phytochromes a, B1 and B2. The Plant Journal, 25, 14.

Yang, Y.J., Zuo, Z.C., Zhao, X.Y., Li, X., Klejnot, J., Li, Y. et al. (2008) Bluelight-independent activity of Arabidopsis cryptochromes in the regulation of steady-state levels of protein and mRNA expression. Molecular Plant, 1, 167-177.

Yang, Z., Liu, B., Su, J., Liao, J., Lin, C. \& Oka, Y. (2017) Cryptochromes orchestrate transcription regulation of diverse blue light responses in plants. Photochemistry and Photobiology, 93, 112-127.

Zeugner, A., Byrdin, M., Bouly, J.P., Bakrim, N., Giovani, B., Brettel, K. et al. (2005) Light-induced electron transfer in Arabidopsis cryptochrome-1 correlates with in vivo function. The Journal of Biological Chemistry, 280, 19437-19440. 
Zhang, T., Maruhnich, S.A. \& Folta, K.M. (2011) Green light induces shade avoidance symptoms. Plant Physiology, 157, 1528-1536.

\section{SUPPORTING INFORMATION}

Additional supporting information may be found in the online version of the article at the publisher's website.
How to cite this article: Zhang, X., Bisbis, M., Heuvelink, E., Jiang, W. \& Marcelis, L.F.M. (2021) Green light reduces elongation when partially replacing sole blue light independently from cryptochrome 1a. Physiologia Plantarum, 1-10. Available from: https://doi.org/10.1111/ppl.13538 